\title{
Absence of slow particle transport in the many-body localized phase
}

\author{
David J. Luitz $\odot^{1, *}$ and Yevgeny Bar Lev $\odot^{2, \dagger}$ \\ ${ }^{1}$ Max Planck Institute for the Physics of Complex Systems, Noethnitzer Str. 38, Dresden, Germany \\ ${ }^{2}$ Department of Physics, Ben-Gurion University of the Negev, Beer-Sheva 84105, Israel
}

(Received 7 August 2020; revised 21 August 2020; accepted 21 August 2020; published 10 September 2020)

\begin{abstract}
We analyze the saturation value of the bipartite entanglement and number entropy starting from a random product state deep in the many-body localized (MBL) phase. By studying the probability distributions of these entropies we find that the growth of the saturation value of the entanglement entropy stems from a significant reshuffling of the weight in the probability distributions from the bulk to the exponential tails. In contrast, the probability distributions of the saturation value of the number entropy are converged with system size, and exhibit a sharp cutoff for values of the number entropy which correspond to one particle fluctuating across the boundary between the two halves of the system. Our results therefore rule out slow particle transport deep in the MBL phase and confirm that the slow entanglement entropy production stems uniquely from configurational entanglement.
\end{abstract}

DOI: 10.1103/PhysRevB.102.100202

Introduction. Generic interacting quantum many-body systems are expected to thermalize after a quench by virtue of the eigenstate thermalization hypothesis [1-7]. However, the addition of sufficiently strong quenched disorder allows such systems to avoid thermalization [8-13], a phenomenon which is called many-body localization (MBL). For one-dimensional systems the existence of the MBL phase at strong disorder is now firmly established [14], but the situation for higherdimensional systems is still an open question [15-18]. When all many-body eigenstates are localized, the phenomenology of MBL is understood by the emergence of local conserved quantities called the 1-bits $[9,19,20]$. The existence of 1-bits predicts the absence of particle transport and thermalization in the MBL phase, but also an unbounded logarithmic growth of the bipartite entanglement entropy [9,19,21-25] up to a nonthermal, extensive, saturation value. This behavior is in stark contrast with the thermal phase, where the entanglement entropy grows as a power law in time [26,27]. Without disorder, a linear growth of the entanglement entropy is typically observed [28].

Within the 1-bit model the production of entanglement deep in the MBL phase does not rely on particle transport, since due to the emergent conservation laws the particle number in any part of the system is essentially constant for all times [29]. This view was challenged very recently in a study of the entropy of the subsystem particle number distribution

\footnotetext{
*dluitz@pks.mpg.de

†ybarlev@bgu.ac.il
}

[30], where a long growth regime of the number entropy was observed, which was argued to continue indefinitely in the thermodynamic limit in the MBL phase, suggesting that there is very slow transport in the MBL phase.

In the present work, we address this question by a detailed statistical analysis of the behavior of the saturation values of the entanglement and the entropy of the subsystem particle number distribution (number entropy). We find that the fluctuations of the particle number are strictly limited at strong disorder and preclude an indefinite growth of the number entropy.

Model and method. We consider the standard model of many-body localization, an open spin chain with random fields:

$$
\hat{H}=J \sum_{i=1}^{L-1} \boldsymbol{S}_{i} \cdot \boldsymbol{S}_{i+1}+\sum_{i=1}^{L} h_{i} \hat{S}_{i}^{z},
$$

where $J$ corresponds to the interaction between the spins and the random fields are drawn from a box distribution with $h_{i} \in[-W, W]$. Without loss of generality we set $J=$ 1 throughout this work. Using the Jordan-Wigner transformation, this model maps exactly to a model of interacting spinless fermions,

$$
\begin{aligned}
\hat{H}= & \frac{J}{2}\left(\hat{c}_{i}^{\dagger} \hat{c}_{i+1}+\hat{c}_{i+1}^{\dagger} \hat{c}_{i}\right)+J \sum_{i=1}^{L-1}\left(\hat{n}_{i}-\frac{1}{2}\right)\left(\hat{n}_{i+1}-\frac{1}{2}\right) \\
& +\sum_{i=1}^{L} h_{i}\left(\hat{n}_{i}-\frac{1}{2}\right),
\end{aligned}
$$

where $\hat{c}_{i}^{\dagger}$ creates a fermion at site $i$ and $\hat{n}_{i}=\hat{c}_{i}^{\dagger} \hat{c}_{i}$. The model conserves the total magnetization (respectively, the particle number), and throughout this work we fix $\sum_{i} \hat{S}_{i}^{z}=0$ (respectively, half-filling). While the model (1) has been studied in great detail, the critical disorder is only known with a large 
margin of error, $W_{c}=3.8 \pm 1$ [22,31-34]. We therefore focus on quite strong disorder strengths to be sufficiently far from the critical regime, which is known to exhibit strong finite size effects [35-37].

We study the behavior of the system at effectively infinite times using full diagonalization of the Hamiltonian (1). We extract eigenstates and eigenvalues to evolve the initial product states $\left|\sigma_{1}, \sigma_{2}, \ldots, \sigma_{L}\right\rangle$ in time. Here, $\sigma_{i}= \pm \frac{1}{2}$ are the eigenvalues of the corresponding local $\hat{S}_{i}^{z}$ operators. The initial states have a definite number of up spins (i.e., $\sigma_{i}=+1 / 2$ ) in any subsystem, which corresponds to a definite number of particles in the equivalent spinless fermion model (2).

Results. We consider a quench from a product state $\left|\psi_{0}\right\rangle=$ $\left|\sigma_{1}, \sigma_{2}, \ldots, \sigma_{L}\right\rangle$ in the $\hat{S}_{z}$ basis, and cut the system into two subsystems of equal size, $A$ and $B$, where spins $i=1, \ldots, L / 2$ are in subsystem $A$ and spins $L / 2+1, \ldots, L$ are in subsystem $B$. Due to the conservation law $\sum_{i} \hat{S}_{i}^{z}=0$, the reduced density matrix $\hat{\rho}_{A}(t)=\operatorname{Tr}_{B}|\psi(t)\rangle\langle\psi(t)|$ of the subsystem $A$ is block diagonal with blocks labeled by the number of up spins $n_{A}$ in the subsystem. The probability $p\left(n_{A}\right)$ to find $n_{A}$ up spins (corresponding to particles in the spinless system) in subsystem $A$ is given by the trace of the reduced density matrix $\hat{\rho}_{A}$ in this block.

The entanglement entropy is given by

$$
S_{E}=-\operatorname{Tr}\left(\hat{\rho}_{A} \ln \hat{\rho}_{A}\right)
$$

and the number entropy $S_{N}$ is the Shannon entropy of the number distribution $p\left(n_{A}\right)$ [38]:

$$
S_{N}=-\sum_{n_{A}} p\left(n_{A}\right) \ln p\left(n_{A}\right) .
$$

In the context of MBL it is useful to split the contributions to entanglement from particle number fluctuations and the configurations of the particles, by introducing the configurational entropy $S_{C}$, which is the difference $S_{E}-S_{N}[30,38]$.

The wave function $|\psi(t)\rangle$ of the system evolves according to

$$
|\psi(t)\rangle=\sum_{n} e^{-i E_{n} t}\langle n \mid \psi(0)\rangle|n\rangle,
$$

where $E_{n}$ and $|n\rangle$ are the eigenvalues and eigenstates of the Hamiltonian. At any point in time we can calculate the entanglement entropy and the number entropy $S_{N / E}(t)$, using (3) and (4) and the definition of $\hat{\rho}_{A}$. Since in this work we are interested in the saturation values of the entropies, we consider the infinite time average

$$
S_{N / E}^{\infty}=\lim _{T \rightarrow \infty} \frac{1}{T} \int_{0}^{T} d t S_{N / E}(t),
$$

which is estimated numerically by averaging the entropies over 40 time points at very late times to represent the saturation value of the entropy for finite systems. Practically, we have checked that the saturation values are robustly reached at times as late as $t \in\left[10^{16}, 10^{24}\right]$ for all sizes and disorder strengths which we consider in this work.

The entropies exhibit significant temporal fluctuations at late times (cf. Appendix B), therefore in order to get a more robust insight of the late time behavior, we also consider the

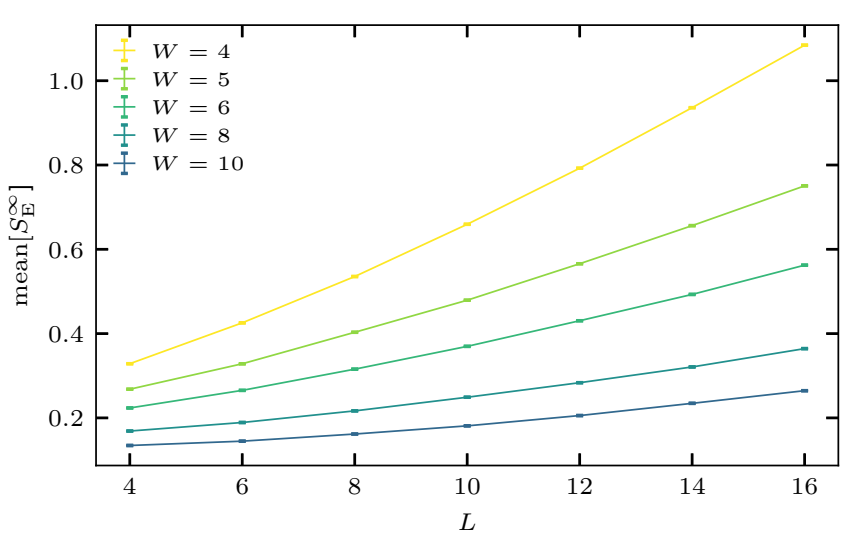

FIG. 1. Disorder average of the long time-averaged entanglement entropy $S_{E}^{\infty}$ as a function of system size $L$ for different disorder strengths $W$.

infinite time average of the number distribution

$$
p^{\infty}\left(n_{A}\right)=\lim _{T \rightarrow \infty} \frac{1}{T} \int_{0}^{T} d t p\left(n_{A}, t\right),
$$

which unlike $S\left[p^{\infty}\left(n_{A}\right)\right]$ is not affected by temporal fluctuations, since it can be calculated numerically exactly. It is easy to show that the entropy $S\left[p^{\infty}\left(n_{A}\right)\right]$ bounds from above the infinite time-averaged number entropy, $S_{N}^{\infty}$, noting that the entropy is a concave function of $p(n)$ and using Jensen's inequality.

In this work we do not study the temporal dependence, but focus directly on the saturation values of the entropies. If the saturation value grows with system size, then the dynamical growth regime continues indefinitely in the thermodynamic limit, and if it is independent of the system size, the temporal growth regime is transient.

In Fig. 1, we show the disorder-averaged saturation value of the entanglement entropy $S_{E}^{\infty}$, obtained by time evolution of the wave function to very long times $t \geqslant 10^{16}$ and averaging over 40 time points for each of the 50 random initial product states in addition to averaging over the disorder realizations. We note that it is important to average over a very large number $(n=50000)$ of disorder realizations to obtain converged statistical averages.

It is clearly visible that the saturation value of the entanglement entropy grows with system size. Interestingly, we observe a significant upturn of the curves even for very strong disorder, which is only weakly visible in previous data obtained at weak interaction strengths $[23,39]$.

The full lines in Fig. 2 show the time-averaged number entropy $S_{N}^{\infty}$ as a function of system size $L$ and for different disorder strengths $W$, spanning both the critical and MBL regimes. While for $W \leqslant 6, S_{N}^{\infty}$ still grows slightly with system size, we observe a saturation and even a weak decrease for strong disorders $(W \geqslant 8)$. This becomes even more apparent if we consider $S\left[p^{\infty}\left(n_{A}\right)\right]$, which satisfies $S_{N}^{\infty} \leqslant S\left[p^{\infty}\left(n_{A}\right)\right]$ and is plotted as the dashed lines in Fig. 2. We see that $S\left[p^{\infty}\left(n_{A}\right)\right]$ is saturated for $W=6$ and slightly decreases with system size for stronger disorders. Since $S_{N}^{\infty}$ cannot exceed $S\left[p^{\infty}\left(n_{A}\right)\right]$, we conclude that $S_{N}^{\infty}$ is independent of the system size for strong disorder. 


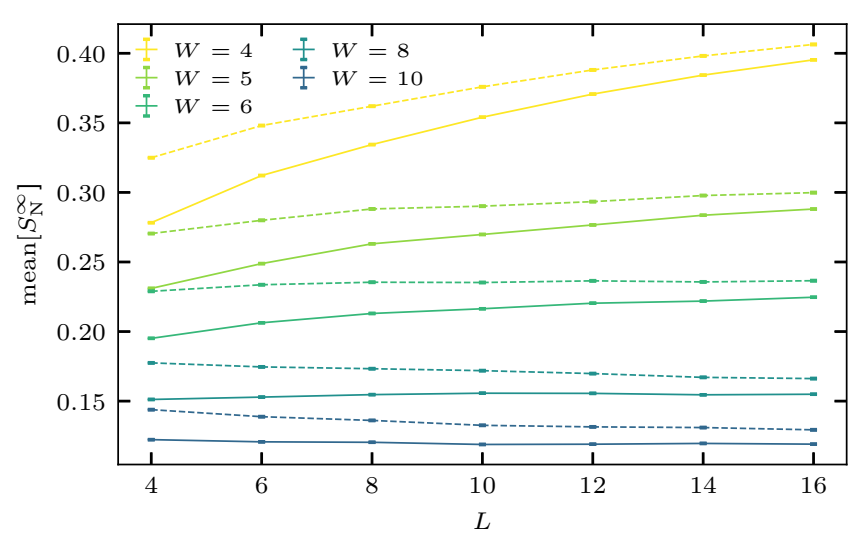

FIG. 2. Full lines: Disorder average of the time-averaged number entropy $S_{N}^{\infty}$ as a function of system size $L$ for different disorder strengths $W$. Dashed lines: Disorder average of the entropy of the infinite time-averaged subsystem number distribution $S\left[p^{\infty}\left(n_{A}\right)\right]$, which is an upper bound of $S_{N}^{\infty}$.

As the mean contains only limited information about the probability distribution, we study the full distributions of $S_{E / N}^{\infty}$. Figure 3 shows the distribution of $S_{E}^{\infty}$ over 50 random initial product states and 50000 disorder realizations. For

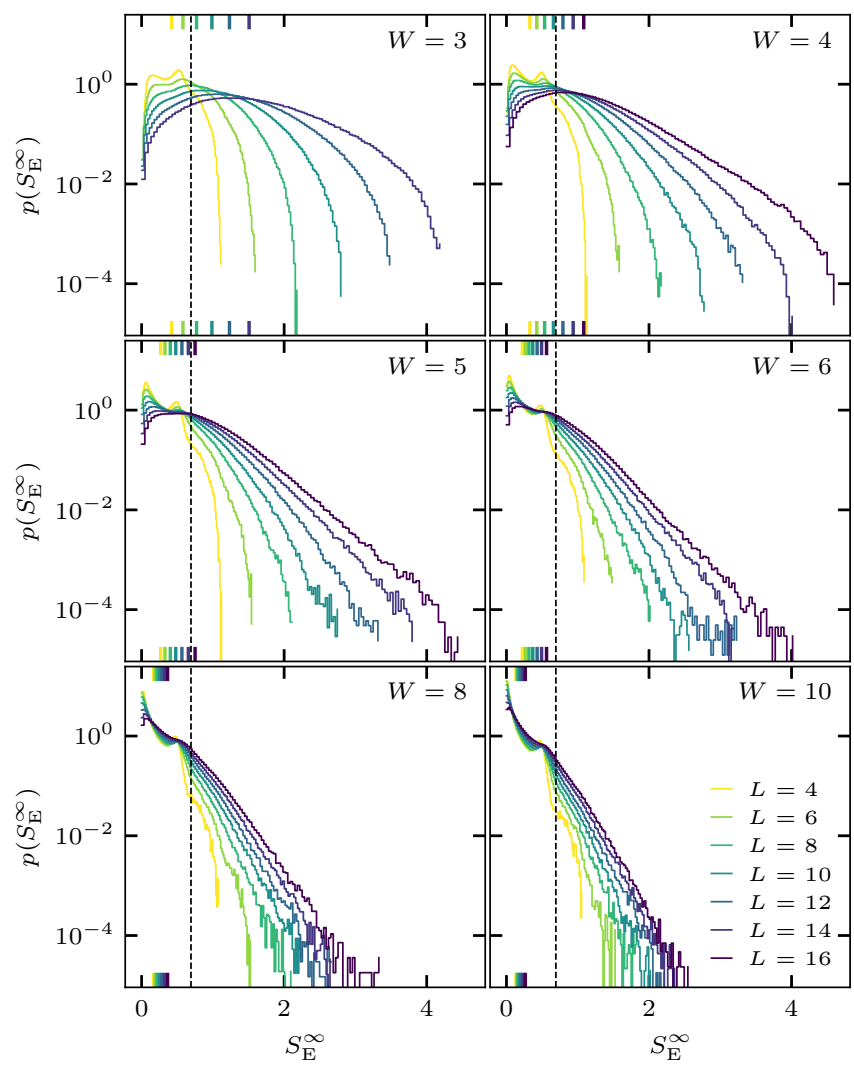

FIG. 3. Distribution of the time-averaged entanglement entropy $S_{E}^{\infty}$ for disorder strengths $W=3,4,5,6,8,10$ and different system sizes $L$. The distributions are taken over 50000 disorder realizations and 50 initial random product states. For each disorder realization the time average is computed by sampling 40 time points for $t \geqslant 10^{16}$. The black dashed line shows the entropy $S_{E}=\ln (2)$ and the colored ticks on the horizontal axis mark the corresponding mean of $S_{E}^{\infty}$.

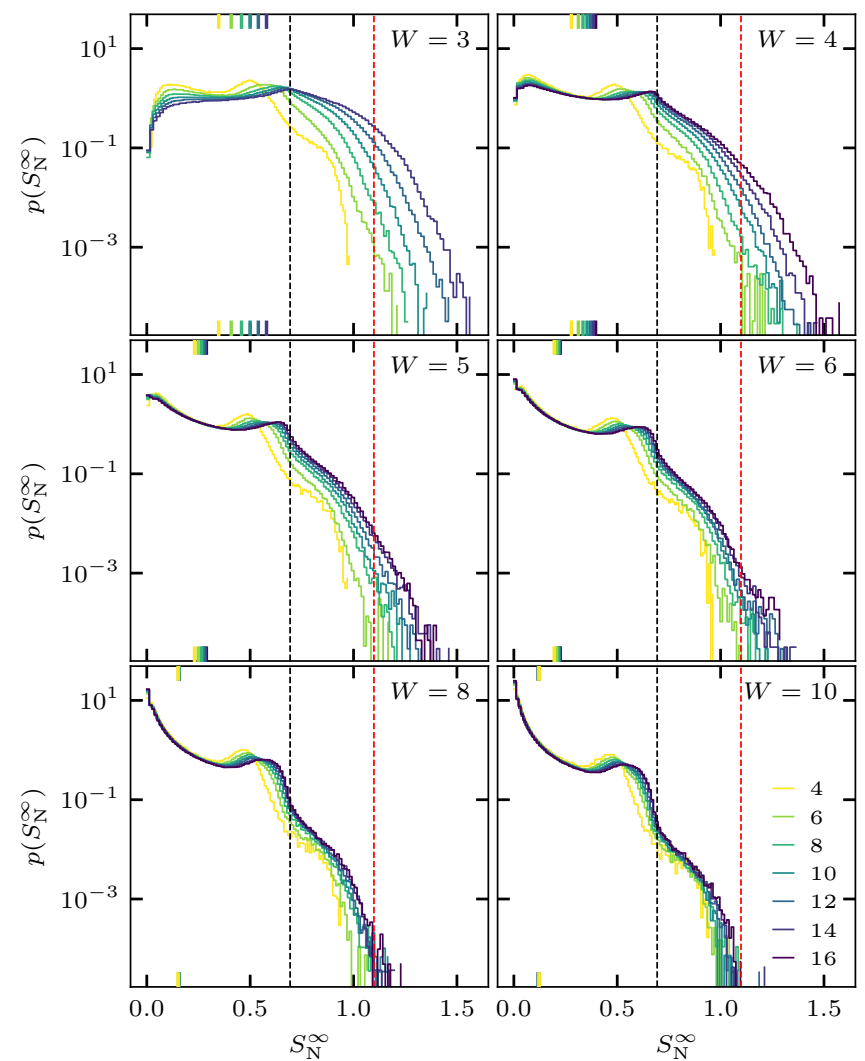

FIG. 4. Similarly to Fig. 3, but for the time-averaged number entropy $S_{N}^{\infty}$. The red dashed line shows the entropy $S=\ln (3)$.

intermediate disorder strengths, in the critical regime $W=$ $3,4,5$ for our system sizes, the distributions of the entanglement entropy are very broad and the growth of the mean is clearly visible in a reshuffling of the weight from low to high entropy as the system size is increased. It is interesting to see that the low entropy part of the distribution of $S_{E}^{\infty}$ is significantly different from the distribution of the entanglement entropy of eigenstates [cf., e.g., Figs. 10(c) and 10(d) in Ref. [40]]. While the mean entanglement of eigenstates does not depend on the system size [41], $S_{E}^{\infty}$ grows with size as shown in Fig. 1, due to dephasing between the various eigenstates which are spanning the initial state.

At very strong disorder, the weight around $S_{E}=0$ is visibly decreasing with increasing systems size, which leads to a corresponding increase in the mean. For high entropies, the distribution exhibits a long, seemingly exponential tail, with a negligible contribution to the mean.

In Fig. 4 we consider the distribution of the timeaveraged number entropy $S_{N}^{\infty}$. While in the critical regime $(W=3,4,5)$ there is a significant reshuffling of the weight from low to high number entropies as the system size is increased, the entire distribution seemingly converges to a limiting distribution at large sizes and strong disorders $W=8,10$. This is accompanied with an effective independence of the mean of the distribution on the system size, as is also shown in Fig. 2. For strong disorder, the distributions exhibit a secondary peak for $S<\ln (2)$, which is reminiscent of the $\ln (2)$ peak in the distributions of the eigenstate entanglement entropy $[40,42,43]$. Here this peak is broadened and stays strictly 


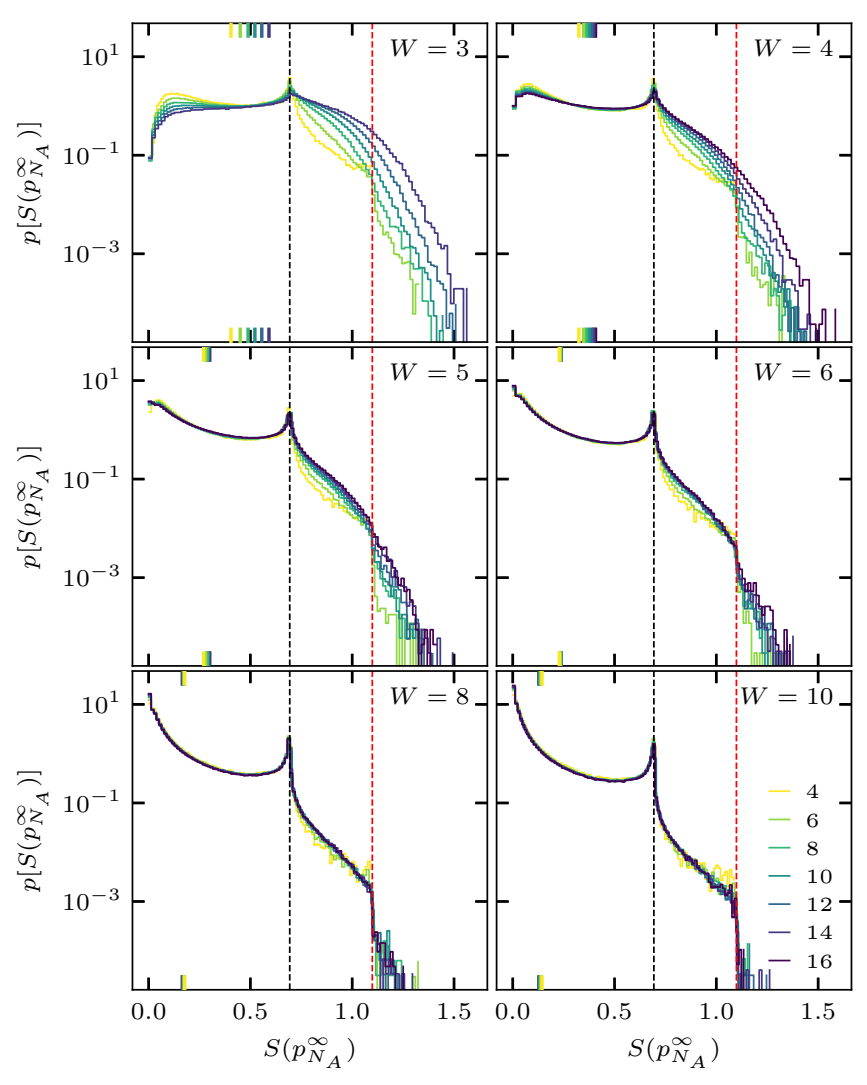

FIG. 5. Similarly to Fig. 4, but for the entropy of the timeaveraged subsystem particle number distribution $S\left[p^{\infty}\left(n_{A}\right)\right]$.

below $\ln$ (2) (black dashed horizontal line) for all considered system sizes. The observed probability distribution seems to decay significantly for entropies larger than $\ln (3)$ (red dashed horizontal line) as we will discuss in more detail below.

For a better, quantitative understanding of the high entropy part of the distribution of $S_{N}^{\infty}$, we consider next the distributions of $S\left[p^{\infty}\left(n_{A}\right)\right]$, which is cleaner due to the fact that the infinite time average can be calculated exactly and since it provides an upper bound to $S_{N}^{\infty}$. Figure 5 shows the distribution of $S\left[p^{\infty}\left(n_{A}\right)\right]$, which exhibit a sharp secondary peak at $\ln (2)$, corresponding to an equal probability to have $n_{A}, n_{A}+1$ or $n_{A,} n_{A}-1$ particles. Even more interestingly, we observe a steep decrease in the probability density at $\ln (3)$ (red dashed line), which corresponds to an equal probability to measure $n_{A}, n_{A}+1$, or $n_{A}-1$ where $n_{A}$ is the particle number in the initial state. This means that in this rare state one particle has crossed the boundary between the two subsystems.

For strong disorder $(W=8,10)$, we see that the distribution is completely converged with the system size, exhibiting a sharp cutoff at $\ln (3)$. The mean of this distribution does not grow with the system size.

Discussion. In this work we presented a detailed study of the saturation value of entanglement and number entropies including the probability distributions over the initial product states and disorder realizations. We have shown that at strong disorder the mean of the saturation value of the entanglement entropy grows with system size, which is consistent with previous literature. The distributions of these quantities are quite broad and require a very large number of disorder realizations to be sampled precisely. We identify that the growth of the mean with system size results from a reshuffling of weight from low to high entropies.

The entanglement entropy can be decomposed into a sum of the number entropy and the configurational entropy. We show that at strong disorder $W=8,10$ the mean of the saturation value of the number entropy does not grow with system sizes; moreover, for large systems the entire probability distribution converges to a limiting distribution. We further study the entropy of the infinite-time-averaged number distributions, which allows us to show that the number fluctuations are typically bounded by a change of one particle across the two halves of the system. This leads to a sharp cutoff in the probability distribution of the number entropy at $\ln (3)$. Even for the very large number of disorder realizations used in this work, we did not observe realizations with number entropies which exceed this limit, which leads us to conclude that there is no particle transport for sufficiently strong disorder, and the observed growth of the number entropy in time in Ref. [30] is pertinent to the critical regime and likely disappears for stronger disorder or larger system sizes.

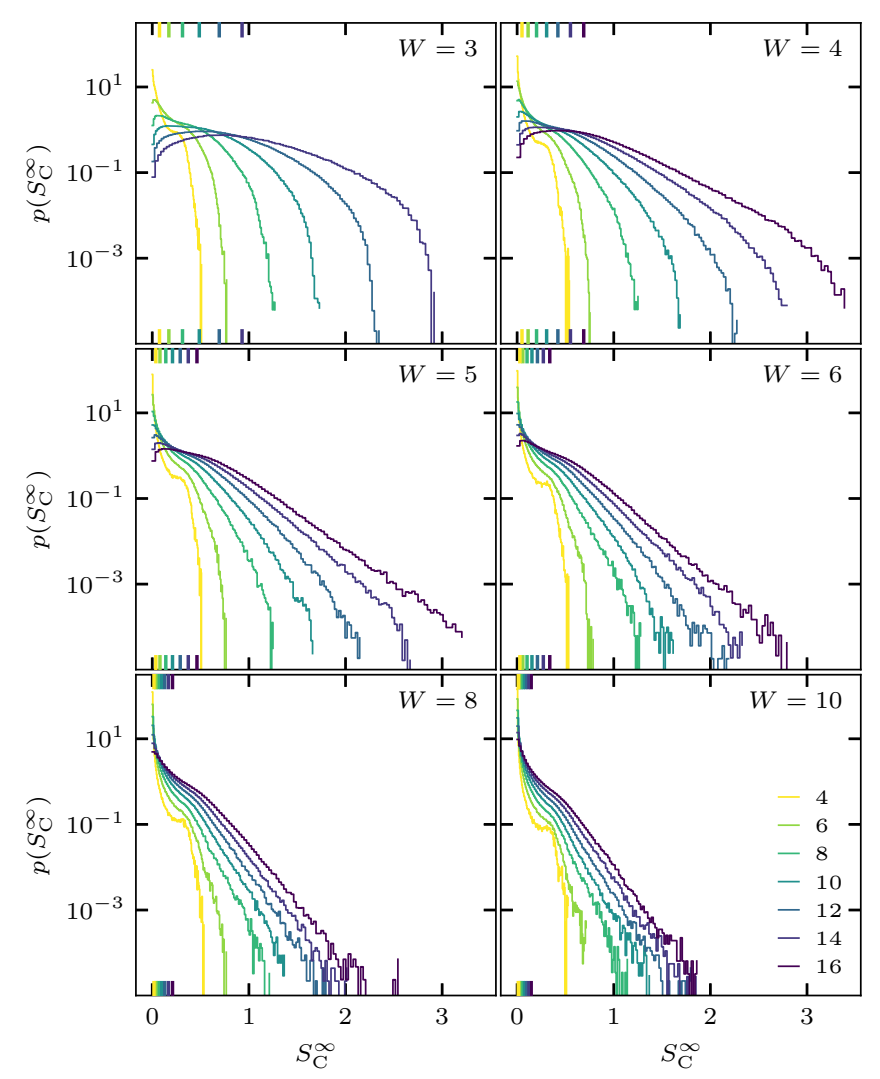

FIG. 6. Distribution of the saturation value of the configurational entropy for disorder strengths $W=3,4,5,6,8,10$ and different system sizes $L$. Distributions are taken over 50000 disorder realizations and 50 initial random product states. In each disorder realization, the time average is sampled over 40 time points for $t \geqslant 10^{16}$. 
This article is an open data publication and the raw data is available as a standard HDF5 file [44].

Acknowledgments. We are grateful to Achilleas Lazarides and Roderich Moessner for useful comments and thank Jesko Sirker for discussions. We acknowledge financial support from the Deutsche Forschungsgemeinschaft through SFB 1143 (Project ID 247310070). Y.B. acknowledges support by the Israel Science Foundation (Grants No. 527/19 and No. 218/19).

\section{APPENDIX A: DISTRIBUTION OF THE CONFIGURATIONAL ENTROPY}

In this Appendix, we provide additional data for the distribution of the saturation value of the configurational entropy $S_{C}^{\infty}$, which is defined as

$$
S_{C}^{\infty}=S_{E}^{\infty}-S_{N}^{\infty} .
$$

We show the full probability distribution (with its mean indicated by colored ticks at the top and bottom of each panel) in Fig. 6. The mean of the distribution grows with system size for all disorder strengths with a slower growth at strong disorder. This growth stems from reshuffling of the weight from low to high entropies.

The distribution of the configurational entropy is quite similar to that of the entanglement entropy and exhibits an exponential tail as well as a peak at zero entropy which decreases with system size. The sharp peak located at $S_{E}^{\infty}<$ $\ln (2)$ for small systems in the entanglement entropy distribution is strongly suppressed in the number entropy distribution, indicating that it stems from the number entropy.

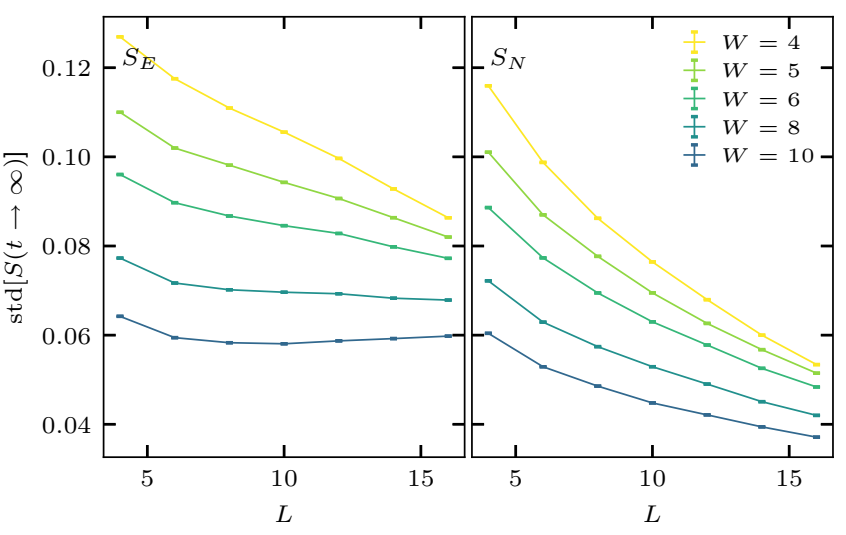

FIG. 7. Temporal fluctuations of the entanglement entropy $S_{E}$ (left) and the number entropy $S_{N}$ (right), as a function of the system size and for various disorder strengths.

\section{APPENDIX B: TEMPORAL FLUCTUATIONS OF THE SATURATION VALUE OF THE ENTANGLEMENT AND NUMBER ENTROPIES}

Besides the time average of the late time entropies, we consider also the temporal fluctucations around the saturation value of the entropies in Fig. 7.

While at strong disorder the fluctuations are generally suppressed, it is interesting to see that the fluctuations of the entanglement entropy are larger and do not decay with system size, while the number entropy fluctuations decrease with systems size for all disorder strengths.
[1] M. Feingold, N. Moiseyev, and A. Peres, Ergodicity and mixing in quantum theory. II, Phys. Rev. A 30, 509 (1984).

[2] J. M. Deutsch, Quantum statistical mechanics in a closed system, Phys. Rev. A 43, 2046 (1991).

[3] M. Srednicki, Chaos and quantum thermalization, Phys. Rev. E 50, 888 (1994).

[4] M. Srednicki, The approach to thermal equilibrium in quantized chaotic systems, J. Phys. A: Math. Gen. 32, 1163 (1999).

[5] M. Rigol, V. Dunjko, and M. Olshanii, Thermalization and its mechanism for generic isolated quantum systems, Nature (London) 452, 854 (2008).

[6] L. D'Alessio, Y. Kafri, A. Polkovnikov, and M. Rigol, From quantum chaos and eigenstate thermalization to statistical mechanics and thermodynamics, Adv. Phys. 65, 239 (2016).

[7] F. Borgonovi, F. M. Izrailev, L. F. Santos, and V. G. Zelevinsky, Quantum chaos and thermalization in isolated systems of interacting particles, Phys. Rep. 626, 1 (2016).

[8] D. M. Basko, I. L. Aleiner, and B. L. Altshuler, Metal-insulator transition in a weakly interacting many-electron system with localized single-particle states, Ann. Phys. 321, 1126 (2006).

[9] D. A. Huse, R. Nandkishore, and V. Oganesyan, Phenomenology of fully many-body-localized systems, Phys. Rev. B 90, 174202 (2014).

[10] D. J. Luitz and Y. Bar Lev, The ergodic side of the many-body localization transition, Ann. Phys. 529, 1600350 (2017).
[11] D. A. Abanin and Z. Papić, Recent progress in many-body localization, Ann. Phys. 529, 1700169 (2017).

[12] F. Alet and N. Laflorencie, Many-body localization: An introduction and selected topics, C. R. Phys. 19, 498 (2018).

[13] D. A. Abanin, E. Altman, I. Bloch, and M. Serbyn, Colloquium: Many-body localization, thermalization, and entanglement, Rev. Mod. Phys. 91, 021001 (2019).

[14] J. Z. Imbrie, On many-body localization for quantum spin chains, J. Stat. Phys. 163, 998 (2016).

[15] T. B. Wahl, A. Pal, and S. H. Simon, Signatures of the manybody localized regime in two dimensions, Nat. Phys. 15, 164 (2019).

[16] H. Théveniaut, Z. Lan, and F. Alet, Many-body localization transition in a two-dimensional disordered quantum dimer model, Phys. Rev. Res. 2, 033154 (2020).

[17] Jae-yoon Choi, S. Hild, J. Zeiher, P. Schauß, A. Rubio-Abadal, T. Yefsah, V. Khemani, D. A. Huse, I. Bloch, and C. Gross, Exploring the many-body localization transition in two dimensions, Science 352, 1547 (2016).

[18] E. Chertkov, B. Villalonga, and B. K. Clark, Numerical evidence for many-body localization in two and three dimensions, arXiv:2007.02959.

[19] M. Serbyn, Z. Papić, and D. A. Abanin, Local Conservation Laws and the Structure of the Many-Body Localized States, Phys. Rev. Lett. 111, 127201 (2013). 
[20] J. Z. Imbrie, V. Ros, and A. Scardicchio, Local integrals of motion in many-body localized systems, Ann. Phys. 529, 1600278 (2017).

[21] G. D. Chiara, S. Montangero, P. Calabrese, and R. Fazio, Entanglement entropy dynamics of Heisenberg chains, J. Stat. Mech. (2006) P03001.

[22] M. Žnidarič, T. Prosen, and P. Prelovšek, Many-body localization in the Heisenberg $X X Z$ magnet in a random field, Phys. Rev. B 77, 064426 (2008).

[23] J. H. Bardarson, F. Pollmann, and J. E. Moore, Unbounded Growth of Entanglement in Models of Many-Body Localization, Phys. Rev. Lett. 109, 017202 (2012).

[24] A. Nanduri, H. Kim, and D. A. Huse, Entanglement spreading in a many-body localized system, Phys. Rev. B 90, 064201 (2014).

[25] M. Žnidarič, Entanglement in a dephasing model and manybody localization, Phys. Rev. B 97, 214202 (2018).

[26] D. J. Luitz, N. Laflorencie, and F. Alet, Extended slow dynamical regime close to the many-body localization transition, Phys. Rev. B 93, 060201(R) (2016).

[27] T. L. M. Lezama and D. J. Luitz, Power-law entanglement growth from typical product states, Phys. Rev. Res. 1, 033067 (2019).

[28] H. Kim and D. A. Huse, Ballistic Spreading of Entanglement in a Diffusive Nonintegrable System, Phys. Rev. Lett. 111, 127205 (2013).

[29] J. Hauschild, F. Heidrich-Meisner, and F. Pollmann, Domainwall melting as a probe of many-body localization, Phys. Rev. B 94, 161109(R) (2016).

[30] M. Kiefer-Emmanouilidis, R. Unanyan, M. Fleischhauer, and J. Sirker, Evidence for Unbounded Growth of the Number Entropy in Many-Body Localized Phases, Phys. Rev. Lett. 124, 243601 (2020).

[31] A. Pal and D. A. Huse, Many-body localization phase transition, Phys. Rev. B 82, 174411 (2010).

[32] D. J. Luitz, N. Laflorencie, and F. Alet, Many-body localization edge in the random-field Heisenberg chain, Phys. Rev. B 91, 081103(R) (2015).
[33] T. Devakul and R. R. P. Singh, Early Breakdown of Area-Law Entanglement at the Many-Body Delocalization Transition, Phys. Rev. Lett. 115, 187201 (2015).

[34] E. V. H. Doggen, F. Schindler, K. S. Tikhonov, A. D. Mirlin, T. Neupert, D. G. Polyakov, and I. V. Gornyi, Many-body localization and delocalization in large quantum chains, Phys. Rev. B 98, 174202 (2018).

[35] V. Khemani, S. P. Lim, D. N. Sheng, and D. A. Huse, Critical Properties of the Many-Body Localization Transition, Phys. Rev. X 7, 021013 (2017).

[36] F. Weiner, F. Evers, and S. Bera, Slow dynamics and strong finite-size effects in many-body localization with random and quasiperiodic potentials, Phys. Rev. B 100, 104204 (2019).

[37] D. J. Luitz, I. Khaymovich, and Y. Bar Lev, Multifractality and its role in anomalous transport in the disordered $\mathrm{XXZ}$ spinchain, SciPost Phys. Core 2, 006 (2020).

[38] A. Lukin, M. Rispoli, R. Schittko, M. E. Tai, A. M. Kaufman, S. Choi, V. Khemani, J. Léonard, and M. Greiner, Probing entanglement in a many-body-localized system, Science $\mathbf{3 6 4}$, 256 (2019).

[39] M. Serbyn, Z. Papić, and D. A. Abanin, Universal Slow Growth of Entanglement in Interacting Strongly Disordered Systems, Phys. Rev. Lett. 110, 260601 (2013).

[40] D. J. Luitz, Long tail distributions near the many-body localization transition, Phys. Rev. B 93, 134201 (2016).

[41] X. Yu, D. J. Luitz, and B. K. Clark, Bimodal entanglement entropy distribution in the many-body localization transition, Phys. Rev. B 94, 184202 (2016).

[42] B. Bauer and C. Nayak, Area laws in a many-body localized state and its implications for topological order, J. Stat. Mech. (2013) P09005.

[43] S. P. Lim and D. N. Sheng, Many-body localization and transition by density matrix renormalization group and exact diagonalization studies, Phys. Rev. B 94, 045111 (2016).

[44] D. J. Luitz and Y. Bar Lev, Max Planck Society (2020), https: //dx.doi.org/10.17617/3.4i. 